OPEN ACCESS

Edited by:

Alessandro Marcello,

International Centre for Genetic

Engineering and Biotechnology, Italy

Reviewed by:

Molalegne Bitew,

Ethiopian Biotechnology Institute

(EBTi), Ethiopia

Miroslav Petrovec,

University of Ljubljana, Slovenia

*Correspondence:

Soegianto All

soegianto.ali@atmajaya.ac.id

${ }^{\text {t}}$ These authors have contributed equally to this work and share first authorship

Specialty section:

This article was submitted to

Virus and Host,

a section of the journal

Frontiers in Cellular and

Infection Microbiology

Received: 06 April 2021

Accepted: 18 June 2021

Published: 18 August 2021

Citation:

Mahendra C, Kaisar MMM Vasandani SR, Surja SS, Tjoa E,

Chriestya F, Junusmin KI, Widowati TA, Irwanto A and Ali S (2021) Wide Application of Minimally

Processed Saliva on Multiple RTqPCR Kits for SARS-CoV-2

Detection in Indonesia.

Front. Cell. Infect. Microbiol. 11:691538.

doi: 10.3389/fcimb.2021.691538

\section{Wide Application of Minimally Processed Saliva on Multiple RT-qPCR Kits for SARS-CoV-2 Detection in Indonesia}

\author{
Caroline Mahendra ${ }^{1+}$, Maria Mardalena Martini Kaisar ${ }^{2+}$, Suraj Rajan Vasandani ${ }^{1+}$, \\ Sem Samuel Surja ${ }^{2}$, Enty Tjoa ${ }^{2}$, Febie Chriestya ${ }^{2,3}$, Kathleen Irena Junusmin ${ }^{1}$, \\ Tria Asri Widowati ${ }^{2}$, Astrid Irwanto ${ }^{1,4}$ and Soegianto Ali ${ }^{2 *}$

\begin{abstract}
${ }^{1}$ Nalagenetics Pte Ltd, Singapore, Singapore, ${ }^{2}$ School of Medicine and Health Sciences, Atma Jaya Catholic University of Indonesia, Jakarta, Indonesia, ${ }^{3}$ Rumah Sakit Pendidikan \& Pusat Penelitian Atma Jaya, Jakarta, Indonesia, ${ }^{4}$ Department of Pharmacy, Faculty of Science, National University of Singapore, Singapore, Singapore
\end{abstract}

Saliva as a sample matrix has been an attractive alternative for the detection of SARSCoV-2. However, due to potential variability in collection and processing steps, evaluating a proposed workflow amongst the local population is recommended. Here, we aim to validate the collection and treatment of human saliva as a direct specimen for RT-qPCRbased detection of SARS-CoV-2 in Indonesia. We demonstrated that SARS-CoV-2 target genes were detected in saliva specimens and remained stable for five days either refrigerated or stored at room temperature. The method of processing saliva specimens described in this report bypasses the need for an RNA-extraction process, thereby reducing the cost, time, and manpower required for processing samples. The developed method was tested across nine commercial kits, including the benchmark, to demonstrate its wide applicability on multiple existing workflows. Our developed method achieved an $86 \%$ overall agreement rate compared to paired nasopharyngeal and oropharyngeal swab specimens (NPOP). With the assistance of a saliva sampling device, the collection was found to be more convenient for individuals and improved the overall agreement rate to $97 \%$.

Keywords: saliva, SARS-CoV-2, real-time PCR, direct-PCR, stability, RNA-extraction-free, large capacity, CovID-19

\section{INTRODUCTION}

The first COVID-19 case was reported to the World Health Organization (WHO) on December 31, 2019, and the disease was declared a pandemic on March 11, 2020 (Timeline of WHO's response to COVID-19). COVID-19 is caused by SARS-CoV-2 viral infection, an enveloped virus with a singlestranded positive-strand genomic RNA (Brian and Baric, 2005). One of the modalities used to diagnose COVID-19 is a nucleic acid amplification test (NAAT), including Reverse Transcription quantitative Polymerase Chain Reaction (RT-qPCR), which commonly targets the envelope (E), 
nucleocapsid (N), RNA-dependent RNA polymerase (RdRP), and spike (S) genes (World Health Organization, 2020). Specimens that could be used for NAAT include those obtained from the upper and lower respiratory tracts and gastrointestinal tracts (Wang et al., 2020). Specimens commonly collected from the upper respiratory tracts are nasopharyngeal and oropharyngeal (NPOP) swabs, and those obtained from the lower respiratory tract include bronchoalveolar lavage. The rate of detecting positive infection from bronchoalveolar lavage is superior to that of NPOP swabs; however, it is commonly obtained from inpatients with severe illness or those undergoing mechanical ventilation (Wang et al., 2020). The stool has also been used as a specimen for NAAT methods for SARS-CoV-2 detection, including among children (Zhang et al., 2020).

Collecting NPOP specimens requires trained healthcare personnel and could induce aerosolization, increasing the risk of infection to healthcare personnel. Adequate Personal Protective Equipment (PPE) is needed, and specimen collection can only be done in designated sites to reduce the risk of transmission during the procedure. Indonesia is a very vast country with varying levels of access to collection sites at medical facilities. Patients with suspected infections located far from urban facilities may need to travel on public transport for a period of time to arrive at medical centers for NPOP collection. Such situations could increase the risk of disease spread from the individuals' exposure to the mass population during his/ her travel.

Other studies have shown that saliva can serve as an alternative specimen for NAAT-based SARS-CoV-2 detection (Hung et al., 2020; Wyllie et al., 2020). Its non-invasive nature reduces the level of discomfort experienced when sampling, minimizes production of aerosols, and does not require a trained healthcare provider, which could allow for flexibility of sampling at various collection sites, including at-home. Although there is a reduction in sensitivity to detecting SARS-CoV-2 from saliva specimens, its specificity remained on par with NPOP specimens, suggesting that saliva is still a reliable specimen (Griesemer et al., 2020; Landry et al., 2020; Xu et al., 2020).

The standard protocol for detecting SARS-CoV-2 using the RT-qPCR method from NPOP swabs requires various consumables. The NPOP swab should be immersed in Viral Transport Medium (VTM) for transportation from the collection site to the laboratory and maintained in a cold condition. In the laboratory, the VTM-containing NPOP specimens are extracted to isolate viral RNA. This step generally utilizes a commercial RNA extraction kit, which could take up to $1-1.5$ hours to complete. Purified viral RNA then will be used as a template for RT-qPCR amplification which takes $2-3$ hours from reaction set up to completion. The whole procedure could take $3-4.5$ hours from sample collection to result reporting.

Previous reports have demonstrated comparable results of performing RT-qPCR directly from NPOP without the RNA extraction step (Alcoba-Florez et al., 2020; Smyrlaki et al., 2020). Direct PCR omits the need for RNA extraction kits and reduces the turnover time by $1-1.5$ hours. Currently, in Indonesia, most RNA extraction kits are imported and, particularly during this pandemic, testing kits can be scarce.

Considering the above-mentioned possibilities, we tested and validated the detection of SARS-CoV-2 by RT-qPCR from minimally processed saliva specimens. The validated method would be more convenient for patients, safer for healthcare providers, and reduce the time and cost of the current RTqPCR test to detect COVID-19 infection.

\section{MATERIALS AND METHODS}

\section{Ethical Clearance}

The collection of clinical specimens, NPOP swabs, and saliva specimens were approved by the Institutional Review Board of the School of Medicine and Health Sciences, Atma Jaya Catholic University of Indonesia (No:16/11/KEP-FKIKUAJ/2020).

\section{Study Recruitment}

Recruitment for study participants was done in collaboration with multiple SARS-CoV-2 testing sites. Participants were verbally informed about the study and the procedures involved. Written informed consent was obtained from all participants prior to specimen collection. Inclusion criteria for this study were patients who tested positive up to 14 days before specimen collection or were in close contact with a known positive patient. Exclusion criteria were patients who were critically ill, unconscious, and/or intubated.

\section{Specimen Collection}

Collection of NPOP swabs and saliva specimens were performed for every patient and within one hour of each other. NPOP swabs were collected by a trained medical professional by inserting separate swabs into the participants' nasopharyngeal (NP) and oropharyngeal (OP) cavity and immersed into a single tube containing VTM. Prior to saliva specimen collection, patients were required to satisfy a 30-minute fasting period during which they were prohibited from eating, drinking, smoking, tooth brushing, using mouthwash, and other activities that involved the oral cavity. Unstimulated saliva was collected without the addition of buffers or other stabilizing media into a conical tube (2-5 mL) or a sampling device (0.5-1.0 $\mathrm{mL}$ ) consisting of a cryovial and a mouthpiece (straw or funnel). Specimens were then kept on ice during transport and processed in an enhanced Biosafety Laboratory Level 2 facility at the School of Medicine and Health Sciences, Atma Jaya Catholic University of Indonesia.

\section{Viral RNA Extraction}

Viral RNA was extracted with QIAamp Viral RNA Mini kit (Catalog \# 52906, Qiagen, Hilden Germany) following instructions provided by the manufacturer. $140 \mu \mathrm{L}$ of VTMcontaining NPOP swabs or saliva specimens was mixed with lysis buffer, bound to silica membrane present in the spin column, washed twice, and eluted $(60 \mu \mathrm{L})$ as pure RNA. 


\section{Viral Nucleic Acid Detection With RT-qPCR}

Da An Gene's Detection Kit for 2019 Novel Coronavirus (2019nCoV) RNA (PCR-Fluorescence Probing) (Catalog \# DA-930) was used as the reference nucleic acid detection kit in this study. The RT-qPCR master mix was prepared following the manufacturer's recommended instruction - $17 \mu \mathrm{L}$ of PCR reaction solution $\mathrm{A}$ and $3 \mu \mathrm{L}$ of $\mathrm{PCR}$ reaction solution $\mathrm{B}$ for each reaction. The template $(5 \mu \mathrm{L})$ used was either extracted viral RNA (Viral RNA Extraction), or RNA-extraction-free treated saliva specimens (Validating RNA-Extraction-Free Treatment of Saliva Specimens). Upon template addition, strip tubes were briefly spun down to ensure that all liquid was positioned at the bottom of the tube. Thermocycling conditions were as follows: 15 minutes at $50^{\circ} \mathrm{C}, 15$ minutes at $95^{\circ} \mathrm{C}$, and 45 cycles of 15 seconds at $94^{\circ} \mathrm{C}$ and 45 seconds at $55^{\circ} \mathrm{C}$. The amplification, detection, and analysis were performed using the CFX96 Touch Real-Time PCR detection system (Bio-Rad laboratories). Negative and positive controls were included in each RT-qPCR run. Cycle threshold (Ct) values were analyzed using CFX Maestro software (Bio-Rad laboratories). The Ct-value results represent the amplification cycle in which the fluorescence signal level exceeds the background fluorescence, reflecting the presence of SARS-CoV-2 RNA in the specimen tested. Specimens were interpreted as positive if the cycle threshold (Ct) values for N-gene and ORF1ab were less than 40, and the curve displayed apparent amplification in the typical "S" shaped form. Specimens with no amplification (N/A) or Ct values $>40$ for both genes but with internal control amplified were interpreted as negative. Specimens that only had amplification in one of the target genes, N-gene or ORF1ab but not both, or no amplification at all across all the channels, were interpreted as invalid in this study.

\section{Viability of Saliva as a Sample Matrix}

Paired NPOP and saliva specimens were collected and used to show the viability of saliva as a sample matrix to detect SARSCoV-2 RNA $(n=116)$. Viral RNA from both specimens was extracted with the QIAamp Viral RNA Mini kit (Viral RNA Extraction). Detection of viral RNA from both specimen types was done on our reference kit (Viral Nucleic Acid Detection With $R T-q P C R)$.

\section{Optimization of RNA-Extraction-Free Treatment for Saliva Specimens}

Optimization of RNA-extraction-free treatment of saliva specimens was performed on six previously diagnosed specimens, consisting of positives $(n=3)$ and negatives $(n=3)$. Viral RNA was extracted from NPOP and saliva specimens (Viral RNA Extraction) before being subjected to RT-qPCR to detect SARS-CoV-2 (Viral Nucleic Acid Detection With RT$q P C R$ ) (Figure 1A, Treatments 1 and 2). The remaining saliva specimens were vortexed, aliquoted as $100 \mu \mathrm{L}$ into six $1.5 \mathrm{~mL}$ microtubes, and subjected to the different RNA-extraction-free treatments (Figure 1A, Treatments 3 to 8 ) before being added as templates into the RT-qPCR reaction for viral RNA detection
(Supplementary 1) (Viral Nucleic Acid Detection With $R T-q P C R)$.

The effectiveness of RNA-extraction-free treatment of saliva specimens was evaluated by comparing $\mathrm{Ct}$ values on positive saliva specimens $(n=55)$ that were subjected to RNA extraction Treatment 2 and optimized RNA-extraction-free Treatment 6 (Figure 1A, Supplementary 1).

\section{Saliva Specimen Stability for RNA- Extraction-Free Treatment}

Saliva specimens with Ct values ranging from 14.24 to 32.85 for $\mathrm{N}$-gene and 18.15 to 35.18 for ORF1ab were monitored for specimen stability $(\mathrm{n}=14)$. Collected saliva specimens were aliquoted into $100 \mu \mathrm{L}$ in $1.5 \mathrm{~mL}$ microtubes and stored at room temperature $\left(\sim 25^{\circ} \mathrm{C}\right)$ or inside a refrigerator $\left(2-8^{\circ} \mathrm{C}\right)$. Specimens stored at room temperature were tested daily for five days, while those stored in the fridge were tested on days three through five. A tube from each storage condition was retrieved for each time point, and RNA-extraction-free treatment of saliva specimens (Validating RNA-Extraction-Free Treatment of Saliva Specimens) was carried out before subjecting specimens to RT-qPCR (Viral Nucleic Acid Detection With RT-qPCR).

\section{Validating RNA-Extraction-Free Treatment of Saliva Specimens}

Paired NPOP and saliva specimens were collected and used to assess the performance of our RNA-extraction-free treatment of saliva specimens (Treatment 6; Figure 1A, Supplementary 1) against extracted viral RNA from NPOP specimens $(n=125)$. Collected specimens arriving in the laboratory were stored in a refrigerator at approximately $2-8^{\circ} \mathrm{C}$ and processed within 5 days of the collection date. Viral RNA from NPOP swabs and treated saliva were then used as RT-qPCR templates (Viral Nucleic Acid Detection With RT-qPCR).

\section{Compatibility With Other SARS-CoV-2 RT-qPCR Detection Kits}

We assess the compatibility of this method with eight more commercial kits other than the reference, following instructions provided by each manufacturer. Details of genes targeted, internal control, detection limit, the number of cycles, cycle threshold cut-off, and template volume are summarized in Supplementary 2. Evaluation of each kit was done on specimens previously characterized by our reference kit ( $\mathrm{n}=10$ to 13). The samples selected as a pool to analyze different kits should contain at least two positives. Positive samples selected had target genes detected with $\mathrm{Ct}$ range from 17.07 to 35.54 for $\mathrm{N}$-gene and 18.48 to 37.14 for ORF1ab.

For kits that required internal control to be added into specimens prior to extraction (Fosun and Maccura), the step was modified to add internal control into the RT-qPCR master mix at $0.1 x$ of its recommended volume. The amplification, detection, and analysis were performed using the CFX96 Touch Real-Time PCR detection system and CFX Maestro software (Bio-Rad laboratories). 
A

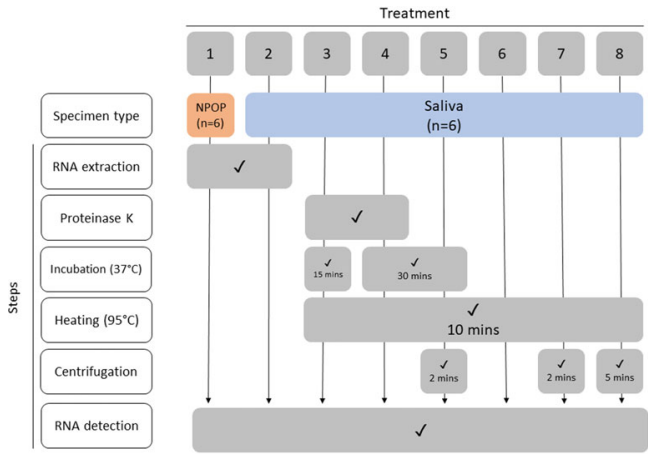

B

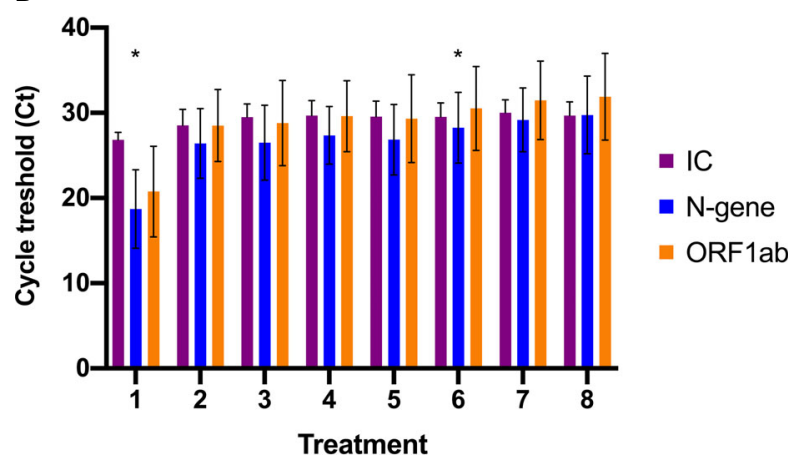

FIGURE 1 | Optimization of RNA-Extraction-free treatment. (A) Flowchart of eight different sample treatments prior SARS-CoV-2 detection (B) Bar graph represents means \pm SEM of three SARS-CoV-2 positive specimens tested for each sample treatment. Asterisk ( ${ }^{*}$ ) denotes the treatments for comparison, where 1 is the comparative method while 6 is the candidate method.

\section{Implementation of a Saliva Collecting Device}

Paired NPOP swab and saliva specimens were collected from collaborating sites during a saliva sampling device implementation at volume $0.5-1.0 \mathrm{~mL}$. The devices used included cryovials with mouthpieces or QuickSpit ${ }^{\mathrm{TM}}$ Collection Kit $(\mathrm{n}=306)$. NPOP swab specimens were collected and underwent RNA extraction following standard procedures performed by the collaborating sites. NPOP results obtained were used as the benchmark for this experiment. The saliva was collected at $0.5-1.0 \mathrm{~mL}$ following instructions for use and processed with RNA-extraction-free treatment before being subjected to RT-qPCR (Viral Nucleic Acid Detection With $R T-q P C R)$.

\section{Data Management and Statistical Analysis}

Collected respondent's information and RT-qPCR data were stored in a Microsoft Excel database with restricted sharing to authors only. Two-by-two contingency matrices were used to compare PCR results from extracted NPOP and test conditions: extracted saliva (Viability of Saliva as a Sample Matrix), treated saliva (Validating RNA-Extraction-Free Treatment of Saliva Specimens), and treated saliva collected with a sampling device (Data Management and Statistical Analysis). Only specimens that returned positive or negative results on both conditions were included in calculating agreement rates. Results were reported as overall agreement, positive percent agreement (PPA), and negative percent agreement (NPA), each with 95\% scores of confidence interval $(95 \% \mathrm{CI})$ calculated using the following formulas:

Overall agreement $(\%)$

$$
\begin{aligned}
= & \frac{\text { positive }_{(\mathrm{NPOP}, \text { tested condition })}+\text { negative }_{(\mathrm{NPOP}, \text { tested condition })}}{\text { total number of specimens }} \\
& \times 100
\end{aligned}
$$

$$
\begin{aligned}
& \operatorname{PPA}(\%)=\frac{\operatorname{positive}_{(\mathrm{NPOP}, \text { tested condition })}}{\text { positive }_{(\mathrm{NPOP}, \text { tested condition })}+\text { positive }_{(\mathrm{NPOP})} \text { negative }_{(\text {tested condition })}} \\
& \mathrm{NPA}(\%)=\frac{\text { negative }_{(\mathrm{NPOP}, \text { tested condition })}}{\text { negative }_{(\mathrm{NPOP}, \text { tested condition })}+\text { negative }_{(\mathrm{NPOP})} \text { positive }_{(\text {tested condition })}} \\
& \times 100
\end{aligned}
$$

Paired two-tailed t-tests were performed to compare the means of Ct value between different saliva specimen treatments (Optimization of RNA-Extraction-Free Treatment for Saliva Specimens) and between the mean difference of the varying saliva storage conditions (Saliva Specimen Stability for RNAExtraction-Free Treatment). The null hypothesis stated there is no difference in means of $\mathrm{Ct}$ value across the tested conditions. Differences with a $p$-value $<0.05$ were considered to be statistically significant. Statistical analysis and data visualization were performed using GraphPad Prism version 8 (GraphPad Software, La Jolla, CA USA) and Microsoft Excel for Windows.

The compatibility of the developed method with different RTqPCR kits was assessed by comparing results obtained on the evaluated kits to those obtained on the reference kit. Results were reported as the rate of invalid specimens, overall agreement, and estimated sensitivity calculated with the following formulas:

$$
\text { Invalid rate }(\%)=\frac{\text { invalid specimens }}{\text { sample size }} \times 100
$$

Overall agreement (\%)

$$
\begin{aligned}
= & \frac{(\text { true positive }+ \text { true negative })}{\text { true positive }+ \text { false positive }+ \text { true negative }+ \text { false negative }} \\
& \times 100
\end{aligned}
$$

Estimated sensitivity $(\%)=\frac{\text { true positive }}{\text { true positive }+ \text { false negative }} \times 100$ 
True positives or negatives were defined as specimens that had concordant results on the evaluated and reference kit. False results were defined as specimens that had discordant results specimens that were positive on the evaluated kit but negative on reference were interpreted as false positives, while specimens that were negative on the evaluated kit but positive on reference were interpreted as false negatives. The specimens that did not return a result on the evaluated kit were defined as invalid.

\section{RESULTS}

\section{Transitioning From NPOP Swab to Saliva for Detection of SARS-CoV-2 by RT-qPCR}

We first sought to validate saliva as a specimen suitable for the detection of SARS-CoV-2 by RT-qPCR. A total of 103 samples returned positive or negative results on both specimens out of the collected 116 samples. We found an $89.3 \%$ overall agreement between specimens extracted from saliva and NPOP, consisting of $84.5 \%$ positive percent agreement (PPA) and $100 \%$ negative percent agreement (NPA) (Table 1, Supplementary 3). Of the specimens, eleven tested negative on saliva but positive on NPOP. This shows the viability of saliva specimens in detecting SARS-CoV-2.

\section{Development of an RNA-Extraction-Free Treatment of Saliva for Detection of SARS-CoV-2}

To streamline sample treatment for downstream RT-qPCR application, we explored several treatment methods on saliva specimens. This included saliva undergoing heating, the addition of Proteinase $\mathrm{K}$, and concentrating by centrifugation paired with RNA extracts from NPOP and saliva as templates for RT-qPCR reaction (Figure 1A, Supplementary 1). We found all treatment methods on saliva specimens resulted in the same qualitative outcome on positive specimens and comparable Ct values for the two target genes (Figure 1B).

Given the need for an affordable and scalable yet effective method, we developed a specimen treatment that only involved the heating of saliva (Figure 1A, Treatment 6). Paired t-test

TABLE 1 | SARS-CoV-2 detection from RNA extracted from NPOP versus saliva sample types.

\begin{tabular}{lccc}
\hline \multirow{2}{*}{ Extracted saliva, $\mathbf{n}$} & \multicolumn{2}{c}{ NPOP } & Total \\
\cline { 2 - 3 } & Positive & Negative & \\
\hline Positive & 60 & 0 & 60 \\
Negative & 11 & 32 & 43 \\
Total & 71 & 32 & 103 \\
& & & \\
Agreements & $\%$ & & $95 \% \mathrm{Cl}$ \\
Overall $^{*}$ & 89.32 & & $81.88-93.93$ \\
Positive $^{* \star}$ & 84.51 & & $74.35-91.12$ \\
Negative $^{* \star *}$ & 100.00 & & $89.28-100.00$ \\
\hline
\end{tabular}

${ }^{*}$ Overall agreement $=((60+32) / 103) \times 100$

${ }^{* *}$ Positive agreement $=(60 / 71) \times 100$.

${ }^{* * *}$ Negative agreement $=(32 / 32) \times 100$ analysis was done between $\mathrm{Ct}$ values obtained from positive specimens extracted from NPOP swabs, Treatment 1, and RNAextraction-free treatment of saliva specimens, Treatment 6. There was no significant difference in detection of $\mathrm{N}$-gene $(p$-value $=0.102)$ and ORF1ab $(p$-value $=0.107)$, demonstrating effectiveness of SARS-CoV-2 detection from heat-treated saliva specimen as compared to RNA extract from NPOP swabs. This method also confirmed qualitative results on negative specimens (Supplementary 4).

We generated a linear regression from positive saliva specimens to demonstrate the effectiveness of RNA-extractionfree treatment of saliva specimens, Treatment 6, compared to those subjected to RNA extraction, Treatment $2(n=55)$. A strong positive correlation in $\mathrm{Ct}$ value for $\mathrm{N}$-gene (coefficient $=1.00$, $\mathrm{R}^{2}=0.929$ ) and ORF1ab (coefficient $=0.966, \mathrm{R}^{2}=0.837$ ) was observed demonstrating that the Treatment 6 is effective for detection of viral RNA in saliva (Figure 2).

\section{SARS-CoV-2 in Saliva Specimens Remained Stable at $4^{\circ} \mathrm{C}$ and Room Temperature (RT)}

To determine the storage condition for saliva specimens that maintain effective treatment and detection of SARS-CoV-2, we monitored specimen stability at two temperatures on previously confirmed positive saliva specimens for five days $(n=14)$. Each specimen was then subjected to heat treatment followed by RTqPCR detection at selected time points during the period of storage. We found that detection of both target genes remained stable for 5 days at both storage conditions for specimens from low to high viral load with initial Ct ranging from 14 to 35 (Figure 3, Supplementary 5).

On detection of $\mathrm{N}$-gene, we found no significant difference in $\mathrm{Ct}$ values upon storage for 5 days at $4^{\circ} \mathrm{C}(\Delta \mathrm{Ct}=0.52$, $p$-value $=$ $0.262)$ and room temperature $(\Delta \mathrm{Ct}=1.00, p$-value $=0.066)$. There was no significant difference in the detection of $\mathrm{N}$-gene between storing specimens at both temperatures ( $p$-value $=$ $0.341)$. ORF1ab detection slightly improved upon storing for a period of 5 days, as seen in the decrease in $\mathrm{Ct}$ value at $4^{\circ} \mathrm{C}(\Delta \mathrm{Ct}=$ -0.386, $p$-value $=0.295)$ and room temperature $(\Delta \mathrm{Ct}=-0.281$, $p$-value $=0.559)$, although there was no significant difference between the two temperatures $(p$-value $=0.671)$. This shows that SARS-CoV-2 remains stable in saliva specimens for up to five days in both storage conditions.

\section{RNA-Extraction-Free Treatment of Saliva Specimens Is Reliable for Detecting SARS-CoV-2}

To assess the performance of the developed method, patients were recruited with written informed consent for collection of NPOP swabs and saliva at the same time point by health care workers. A total of 110 samples returned positive or negative results on both specimens out of the collected 125 samples. NPOP specimens were subjected to QIAamp Viral RNA Extraction, while saliva specimens were treated under the RNA-Extraction-free method, and both were applied as a template for subsequent RT-qPCR detection. We found the 


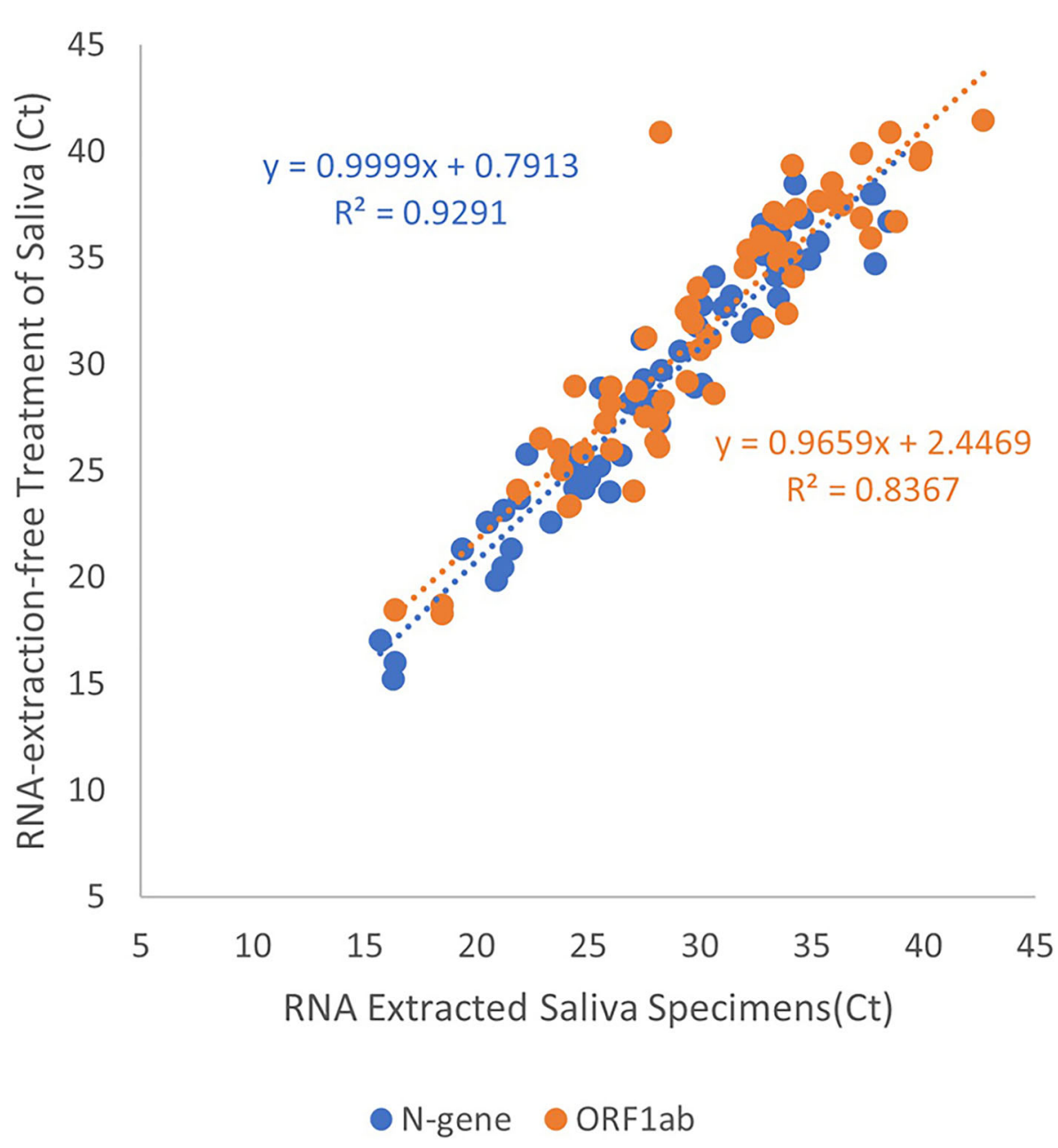

FIGURE 2 | Linear regression of Ct values obtained from adding RNA extracted or heated-only saliva for RT-qPCR template. Data points in blue indicate Ct values for the detection of $\mathrm{N}$-gene while orange for the detection of ORF1ab. Results obtained from extracted RNA from saliva specimens were plotted on the x-axis, with heated-only saliva on the $y$-axis. The line of best fit was plotted for both target genes to display a direct proportion between the two variables.

overall percent agreement between all RNA extract from NPOP swabs and treated saliva specimens to be $86.4 \%$, with $79.2 \%$ PPA and 100\% NPA (Table 2, Supplementary 6). Fifteen specimens tested different results across the two specimen types. This was expected due to variation in specimen types but did not rule out the validity of this method of saliva treatment. Upon closer inspection, treated saliva specimens collected in a saliva sampling device demonstrated better performance than those collected in conical tubes, with an increase of $11.4 \%$ in overall agreement and $13.1 \%$ in overall agreement PPA (Supplementary 7).

\section{Heat-Treated Saliva Specimen Is Versatile With a Number of SARS-CoV-2 RT-qPCR Kits}

To assess the versatility of the developed method for saliva treatment, we subjected the heat-treated saliva specimens $(n=$ 10 to 13) for detection using eight more commercial SARS-CoV2 RT-qPCR kits (Supplementary 2). Results obtained were then compared to the reference kit that this treatment method was developed on, Da An Gene. The parameters of interest to assess the compatibility of the treatment with a commercial kit were their performance at generating invalid results and overall agreement to results produced by the reference kit.

Most of the kits tested generated invalid rates of under $10 \%$, demonstrating that the treatment did not risk inhibiting reactions of other commercial kits, except for Vazyme, which had $40 \%$ invalid rates $(n=4 / 10)$. Two kits obtained results that were in $100 \%$ agreement with the reference, Maccura $(n=11 / 11)$ and Fosun $(n=11 / 11)$. Ardent $(n=10 / 11)$ and SD Biosensor $(n=11 / 12)$ displayed above $90 \%$ agreement, followed by Biosewoom at $80 \%(n=8 / 10)$ and Fortitude $70 \%(n=7 / 10)$. 3S and Vazyme had the lowest agreement to the reference, $40 \%$ $(n=4 / 10)$ and $33 \%(n=2 / 6)$, respectively (Table 3$)$. Upon closer inspection, the lower overall agreement generated by $3 \mathrm{~S}$ and Vazyme $(n=4 / 6)$ was contributed to by the detection of false positives ( $3 S n=6 / 10$; Vazyme $n=4 / 6$; Supplementary 8). This 

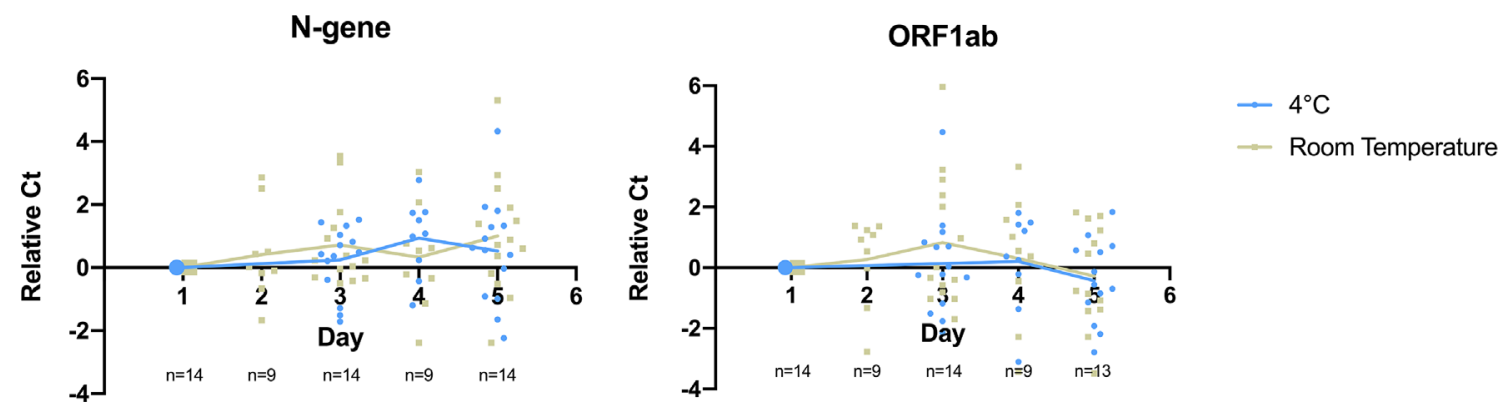

FIGURE 3 | Detection of SARS-CoV-2 target genes N-gene and ORF1ab remained stable for 5 days at cold and room temperature storage conditions. Relative Ct on the $y$-axis plots the difference between Ct value obtained at a given day and Day 1 for the monitored sample. Data points in blue refer to samples stored inside a refrigerator $\left(2-8^{\circ} \mathrm{C}\right)$, while grey refers to samples stored at room temperature. Straight lines display the average relative Ct obtained across storage days.

could arise due to their detection limit being lower than the reference kit, resulting in samples of lower viral load detectable by $3 \mathrm{~S}$ and Vazyme but not by Da An Gene (Supplementary 2). Together, our results demonstrated that most kits were compatible with the developed method for treatment of saliva with varying performance, indicating that validation is recommended before implementing the method with any existing workflow.

\section{Implementation of Saliva Collection and Treatment Method for SARS-CoV-2 Diagnostics}

To better assist with the saliva specimen collection process, we utilized saliva sampling devices for easy and safe sampling of human saliva. The study recruitment was continued at multiple collaborating sites using saliva sampling devices, consisting of cryovials with mouthpieces or QuickSpit ${ }^{\mathrm{TM}}$ Collection kit. Paired NPOP swabs and saliva were collected and processed to evaluate the percent agreements between extracted RNA from NPOP specimens and RNA-extractionfree treatment of saliva specimens collected with a saliva sampling device. A total of 293 samples returned positive or negative results on both specimens out of the collected 306 samples (Table 4, Supplementary 9). The use of a sampling

TABLE 2 | Validation of heated-only saliva as RNA-extraction free treatment.

\begin{tabular}{lccc}
\hline \multirow{2}{*}{ Treated saliva, $\mathbf{n}$} & \multicolumn{2}{c}{ NPOP } & Total \\
\cline { 2 - 3 } & Positive & Negative & \\
\hline Positive & 57 & 0 & 57 \\
Negative & 15 & 38 & 53 \\
Total & 72 & 38 & 110 \\
& & & $95 \% \mathrm{Cl}$ \\
Agreements & $\%$ & & $78.71-91.56$ \\
Overall $^{\star}$ & 86.36 & & $68.43-86.95$ \\
Positive $^{\star \star}$ & 79.17 & & $90.82-100.00$ \\
Negative $^{\star \star *}$ & 100.00 & &
\end{tabular}

${ }^{*}$ Overall agreement $=((57+38) / 110) \times 100$.

${ }^{*}$ Positive agreement $=(57 / 72) \times 100$.

${ }^{* * *}$ Negative agreement $=(38 / 38) \times 100$. device improved the agreement between treated-saliva and extracted-NPOP methods of SARS-CoV-2 treatment before RT-qPCR detection, where 90\% overall agreement was observed with PPA at $85 \%$ and NPA at $100 \%$.

\section{DISCUSSION}

Numerous references have reported the use of human saliva as an attractive specimen for detection of SARS-CoV-2 infection for its practicality in sampling and processing (Azzi et al., 2020; Ott et al., 2020; Iwasaki et al., 2020; Vogels et al., 2020; Watkins et al., 2020; Wyllie et al., 2020; Xu et al., 2020). Utilizing saliva specimens allows for convenient self-collection without the need for a medical professional inserting swabs into a patient's nasopharyngeal and/or oropharyngeal cavity. In this study, we validated the application of human saliva as a candidate specimen for detecting SARS-CoV-2, achieving 90\% agreement with the current conventional specimens - NPOP swabs. Although some discordance was observed between NPOP swabs and saliva specimens, this is in conjunction with previous reports that some variation exists between specimen types (Griesemer et al., 2020; Landry et al., 2020; Vogels et al., 2020; Xu et al., 2020). This could arise from individuals bearing the virus only in their nasopharyngeal cavity but not in their oral cavity. NPOP swabs collect specimen from both respiratory tract sites into a single VTM-containing tube, whereas saliva specimens only collect from the oral cavity. Further investigation on the viral pathway of infection is needed to understand these cases of discordance between specimen types. Results obtained in this study still confirm that saliva is a viable alternative specimen for SARS-CoV-2 detection.

Our developed method could increase the scale of SARSCoV-2 testing since the treatment requires less manpower and time. Furthermore, minimally processed saliva specimens reduce the cost of testing in two ways: 1) exempting the cost of trained professionals for specimen collection and the need for VTM and swabs, and 2) removing the need for the RNA extraction process. Saliva specimen collection could be performed at home and sent to the laboratory in a safe box, hence omitting the need for the 
TABLE 3 | Performance of commercial kits using treated saliva as a template for RT-qPCR.

\begin{tabular}{|c|c|c|c|c|c|}
\hline Commercial kit & Sample size, $\mathbf{n}$ & Invalid specimens, $\mathbf{n}$ & Concordant to reference, $n$ & Invalid rate, \% & Overall agreement, \% \\
\hline Maccura & 11 & 0 & 11 & 0.00 & 100.00 \\
\hline Fosun & 12 & 1 & 11 & 8.33 & 100.00 \\
\hline Ardent & 11 & 0 & 10 & 0.00 & 90.91 \\
\hline SD Biosensor & 13 & 1 & 11 & 7.69 & 91.67 \\
\hline Biosewoom & 11 & 1 & 8 & 9.09 & 80.00 \\
\hline Fortitude & 11 & 1 & 7 & 9.09 & 70.00 \\
\hline $3 S$ & 10 & 0 & 4 & 0.00 & 40.00 \\
\hline Vazyme & 10 & 4 & 2 & 40.00 & 33.33 \\
\hline
\end{tabular}

Rates of the invalid and overall agreement were calculated as compared to results from the reference, Detection Kit for 2019 Novel Coronavirus (2019-nCoV) by Da An Gene Co.

TABLE 4 | Implementation of a sampling device and treatment method for saliva specimens.

\begin{tabular}{|c|c|c|c|}
\hline \multirow{2}{*}{$\begin{array}{l}\text { Treated saliva collected with } \\
\text { sampling device, } n\end{array}$} & \multicolumn{2}{|c|}{ NPOP } & \multirow[t]{2}{*}{ Total } \\
\hline & Positive & Negative & \\
\hline Positive & 45 & 0 & 45 \\
\hline Negative & 8 & 240 & 248 \\
\hline Total & 53 & 240 & 293 \\
\hline Agreements & $\%$ & & $95 \% \mathrm{Cl}$ \\
\hline Overall* & 97.27 & & $94.71-98.61$ \\
\hline Positive $^{\star \star}$ & 84.91 & & $72.95-92.15$ \\
\hline Negative $^{\star \star \star}$ & 100.00 & & $98.43-100.00$ \\
\hline
\end{tabular}

suspected person to travel to the diagnostic center and reduce the chances of transmission during the journey.

The invalid rate for testing saliva was $2 \%$ higher than NPOP specimens (invalid specimens in saliva $=7.8 \%(n=116)$; NPOP $=$ $5.1 \%(n=564))$ (Supplementary 10). This could arise from the carryover of interfering substances from the oral cavity during collection, which was observed in some specimens that were colored, viscous, and/or particulate. Such substances are also potential causes of reaction inhibitors, as seen in the increase of invalid rates from $7 \%(n=116)$ in extracted saliva to $8.8 \%$ $(n=125)$ with treated saliva. The use of a saliva collection device reduced invalid rates on saliva specimens to $3.6 \%$ $(n=306)$ and improved agreements between treated-saliva and extracted NPOP for viral detection. Overall improvement was seen at an increase of $5 \%$ for all agreements from not using a collection device, demonstrating that collecting less volume and using a designated saliva sampling device was more effective. The use of a collection device improved the quality of saliva collected that was visually less viscous, less particulate, and uniformly clear-colored, leading to less accumulation of potential inhibitors.

It is also important to always consider handling precautions during the collection and processing of saliva via heat treatment alone, as the presence of contaminants may inhibit the RT-qPCR reaction. This includes avoiding powdered gloves during collection, transport, and handling, as they are common PCR inhibitors (Lomas et al., 1992; Broyles et al., 2002). Good laboratory practices recommend the use of nitrile gloves in a molecular laboratory (Viana and Wallis, 2011).

One of the highlights of our study is that we also monitored the stability of saliva specimens and found it remained stable at cold $\left(2-8^{\circ} \mathrm{C}\right)$ and room temperature in the laboratory $\left(\sim 25^{\circ} \mathrm{C}\right)$ for up to 5 days. Our finding is concordant with previous studies done by spiking SARS-CoV-2 into saliva specimens (Williams et al., 2021). SARS-CoV-2 may remain stable in saliva despite the presence of RNases in the medium due to its hard outer shell (Goh et al., 2020). This finding is promising for the utilization of saliva specimens in vast countries like Indonesia, where sending specimens from remote areas to diagnostic centers could take days. Nevertheless, we recommend using iceboxes during the transportation of saliva specimens as outside temperatures during the day could be hotter than $30^{\circ} \mathrm{C}$ (WEATHER AND CLIMATE IN INDONESIA | Facts and Details). Once arrived at the laboratory, saliva specimens should also be processed as soon as possible. The sooner the diagnosis is made for the suspected patient; the sooner subsequent measures could be taken, hence limiting the transmission of the disease.

This method could also be used for serial screening of workplaces or schools as frequent NPOP swabbing causes discomfort. The $97 \%$ overall agreement of this method is superior compared to the antigen swab (Mak et al., 2020; Scohy et al., 2020). The developed method of treatment is versatile for several commercial SARS-CoV-2 RT-qPCR kits available in Indonesia. Although there was no particular association observed between the compatibility of saliva undergoing RNA-extraction-free treatment and kits' characteristics, we demonstrated that this method could be adopted with several commercial kits. This allows the implementation of minimally treated saliva specimens across laboratories utilizing various commercial RT-qPCR kits without disrupting their existing workflows, with prior validation of their compatibility highly recommended.

The limitation of this study is that we did not retest the invalid samples for confirmation. Expert guidelines recommend repeating the test process when samples return an invalid result, either with the same workflow or, if available, using an alternate RT-qPCR kit to avoid reporting invalid results (Perhimpunan Dokter Spesialis Patologi Klinik dan Kedokteran Laboratorium Indonesia, 2020). This process of retesting is highly recommended to be implemented with treated saliva as well. Hence a future 


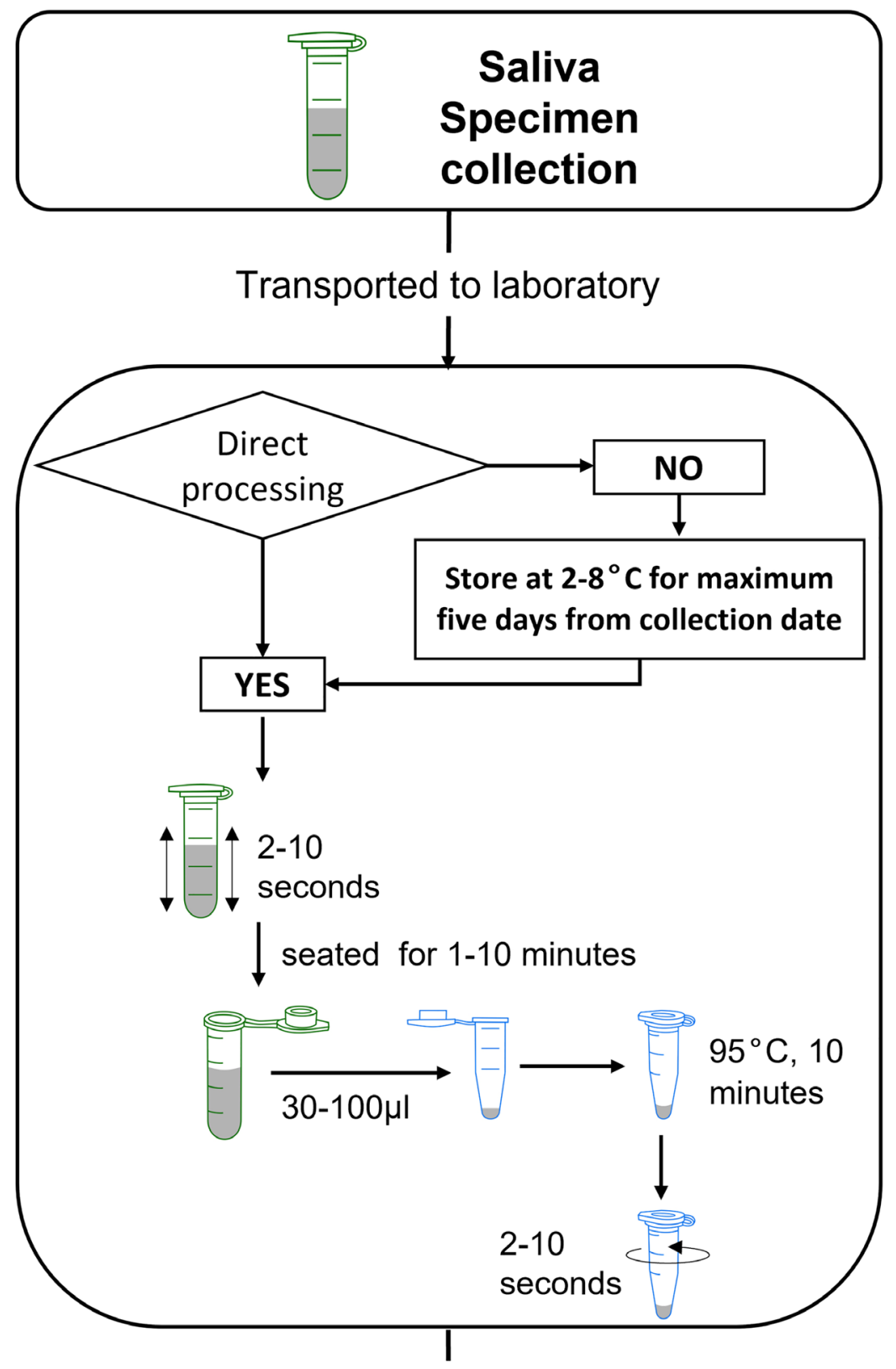

Kept at $2-8^{\circ} \mathrm{C}$, for maximum 2 hours

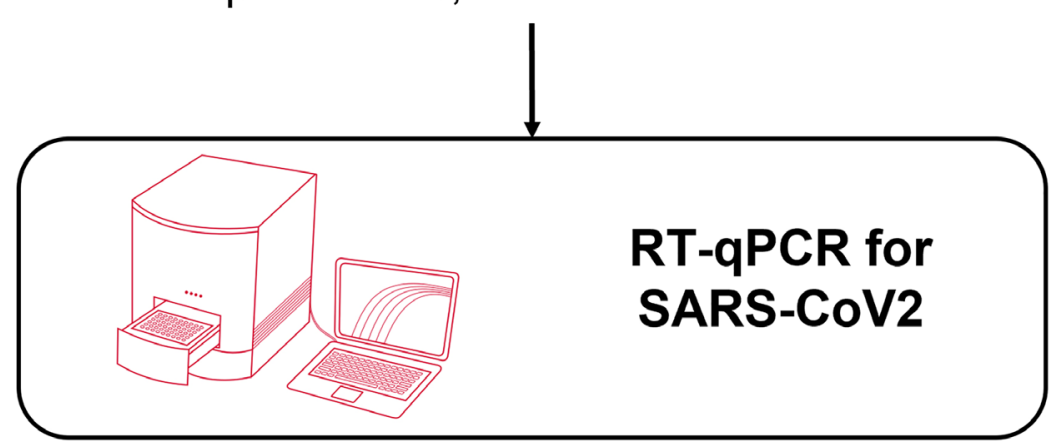

FIGURE 4 | Schematic workflow for collection and treatment of human saliva specimen for RNA-extraction-free detection of SARS-CoV-2 via RT-qPCR. 
study on this would elucidate the cause of discordance between specimen types.

In conclusion, we validated the use of human saliva as a viable alternative specimen to detect SARS-CoV-2 via direct RT-qPCR. Saliva can be collected in a tube without additives and remained stable at cold and room temperatures for five days of storage. Upon arrival in the laboratory, saliva specimens can be treated against the heat incubation method alone, followed by immediate addition as a template for RT-qPCR reaction (Figure 4).

\section{DATA AVAILABILITY STATEMENT}

The raw data supporting the conclusions of this article will be made available by the authors, without undue reservation.

\section{ETHICS STATEMENT}

The studies involving human participants were reviewed and approved by Institutional Review Board of the School of Medicine and Health Sciences, Atma Jaya Catholic University of Indonesia. The patients/participants provided their written informed consent to participate in this study.

\section{AUTHOR CONTRIBUTIONS}

Contributed to study design: CM, SA, MMMK, and SRV. Contributed to patient recruitment: SRV, SS, MMMK, ET, FC, and KJ. Performed laboratory experiments: SV, TW, and MMMK. Contributed to patient data management: SA and CM. Data analysis: CM, SRV, MMMK, and SA. Manuscript writing: CM, SRV, SA, and MMMK. Supervised and conceived

\section{REFERENCES}

Alcoba-Florez, J., González-Montelongo, R., Íñigo-Campos, A., de Artola, D. G.-M., Gil-Campesino, H., Team, T. M. T. S., et al. (2020). Fast SARS-CoV-2 Detection by RT-Qpcr in Preheated Nasopharyngeal Swab Samples. Int. J. Infect. Dis. 97, 66-68. doi: 10.1016/j.ijid.2020.05.099

Azzi, L., Carcano, G., Gianfagna, F., Grossi, P., Gasperina, D. D., Genoni, A., et al. (2020). Saliva Is a Reliable Tool to Detect SARS-CoV-2. J. Infect. 81, e45-e50. doi: 10.1016/j.jinf.2020.04.005

Brian, D. A., and Baric, R. S. (2005). Coronavirus Genome Structure and Replication. Curr. Top. Microbiol. Immunol. 287, 1-30. doi: 10.1007/3-54026765-4_1

Broyles, J. M., O’Connell, K. P., and Korniewicz, D. M. (2002). PCR-Based Method for Detecting Viral Penetration of Medical Exam Gloves. J. Clin. Microbiol. 40, 2725-2728. doi: 10.1128/jcm.40.8.2725-2728.2002

Goh, G. K.-M., Dunker, A. K., Foster, J. A., and Uversky, V. N. (2020). Rigidity of the Outer Shell Predicted by a Protein Intrinsic Disorder Model Sheds Light on the COVID-19 (Wuhan-2019-nCoV) Infectivity. Biomolecules 10 (2), 331. doi: 10.3390/biom 10020331

Griesemer, S. B., van Slyke, G., Ehrbar, D., Strle, K., Yildirim, T., Centurioni, D. A., et al. (2020). Evaluation of Specimen Types and Saliva Stabilization Solutions for SARS-CoV-2 Testing. J. Clin. Microbiol. 59 (5), e01418-20. doi: 10.1128/ JCM.01418-20

Hung, K.-F., Sun, Y.-C., Chen, B.-H., Lo, J.-F., Cheng, C.-M., Chen, C.-Y., et al. (2020). New COVID-19 Saliva-Based Test: How Good Is It Compared With the study: CM, SA, and AI. All authors contributed to the article and approved the submitted version.

\section{FUNDING}

The study was funded by the internal research grant of Atma Jaya Catholic University of Indonesia and Nalagenetics Pte. Ltd. The study was independently designed.

\section{ACKNOWLEDGMENTS}

We would like to thank all the participating healthcare personnel and volunteers of our collaborating sites for contributing to specimen collection: FKIK Atma Jaya, Rumah Sakit Atma Jaya, Rumah Sakit EMC Tangerang, Intibios Lab Mangga Besar, Intibios Lab Pantai Indah Kapuk, and PT Nalagenetik Riset Indonesia. We would also like to acknowledge all patients and respondents who have voluntarily provided their specimen for this study, Sheila Jonnatan for her assistance in the early stage of the laboratory work, COVID-19 Laboratory (AJCUI) for providing the different RT-qPCR kits, and members of PT Nalagenetik Riset Indonesia in assistance for QuickSpit ${ }^{\mathrm{TM}}$ design and development. This work was supported by the COVID-19 Laboratory Center of Atma Jaya Catholic University of Indonesia (AJCUI).

\section{SUPPLEMENTARY MATERIAL}

The Supplementary Material for this article can be found online at: https://www.frontiersin.org/articles/10.3389/fcimb.2021. 691538/full\#supplementary-material

the Current Nasopharyngeal or Throat Swab Test? J. Chin. Med. Assoc. 83, 891-894. doi: 10.1097/JCMA.0000000000000396

Iwasaki, S., Fujisawa, S., Nakakubo, S., Kamada, K., Yamashita, Y., Fukumoto, T., et al. (2020). Comparison of SARS-CoV-2 Detection in Nasopharyngeal Swab and Saliva. J. Infect. 81, e145-e147. doi: 10.1016/j.jinf.2020.05.071

Landry, M. L., Criscuolo, J., and Peaper, D. R. (2020). Challenges in Use of Saliva for Detection of SARS CoV-2 RNA in Symptomatic Outpatients. J. Clin. Virol. 130. doi: $10.1016 /$ j.jcv.2020.104567

Lomas, J., Sunzeri, F., and Busch, M. (1992). False-Negative Results by Polymerase Chain Reaction Due to Contamination by Glove Powder. Transfusion 32, 83 85. doi: 10.1046/j.1537-2995.1992.32192116439.x

Mak, G. C., Cheng, P. K., Lau, S. S., Wong, K. K., Lau, C. S., Lam, E. T., et al. (2020). Evaluation of Rapid Antigen Test for Detection of SARS-CoV-2 Virus. J. Clin. Virol. 129, 104500. doi: 10.1016/j.jcv.2020.104500

Ott, I. M., Strine, M. S., Watkins, A. E., Boot, M., Kalinich, C. C., Harden, C. A., et al. (2020). Simply Saliva: Stability of SARS-CoV-2 Detection Negates the Need for Expensive Collection Devices. medRxiv doi: 10.1101/ 2020.08.03.20165233

Perhimpunan Dokter Spesialis Patologi Klinik dan Kedokteran Laboratorium Indonesia (2020). Panduan Tatalaksana Pemeriksaan Tes Cepat Molekuler (Tcm) Dan Polymerase Chain Reaction (PCR) Sars-cov-2. Available at: https:// www.pdspatklin.or.id/assets/files/pdspatklin_2020_04_30_19_20_35.pdf (Accessed May 25, 2021).

Scohy, A., Anantharajah, A., Bodéus, M., Kabamba-Mukadi, B., Verroken, A., and Rodriguez-Villalobos, H. (2020). Low Performance of Rapid Antigen Detection 
Test as Frontline Testing for COVID-19 Diagnosis. J. Clin. Virol. 129, 104455. doi: 10.1016/j.jcv.2020.104455

Smyrlaki, I., Ekman, M., Lentini, A., Rufino de Sousa, N., Papanicolaou, N., Vondracek, M., et al. (2020). Massive and Rapid COVID-19 Testing Is Feasible by Extraction-Free SARS-CoV-2 RT-PCR. Nat. Commun. 11, 4812. doi: 10.1038/s41467-020-18611-5

Timeline of WHO's Response to COVID-19. Available at: https://www.who.int/ emergencies/diseases/novel-coronavirus-2019/interactive-timeline (Accessed June 14, 2021).

Viana, R. V., and Wallis, C. L. (2011). "Good Clinical Laboratory Practice (GCLP) for Molecular Based Tests Used in Diagnostic Laboratories," in Wide Spectra of Quality Control. Ed. I. Akyar (London United Kingdom: IntechOpen limited). doi: $10.5772 / 23963$

Vogels, C. B. F., Watkins, A. E., Harden, C., Brackney, D. E., Shafer, J., Wang, J., et al. (2020). Salivadirect: A Simplified and Flexible Platform to Enhance SARSCoV-2 Testing Capacity. Med. (N Y) 2 (3), 263-280.e6. doi: 10.1016/ j.medj.2020.12.010

Wang, W., Xu, Y., Gao, R., Lu, R., Han, K., Wu, G., et al. (2020). Detection of SARS-CoV-2 in Different Types of Clinical Specimens. JAMA 323, 1843-1844. doi: $10.1001 /$ jama.2020.3786

Watkins, A. E., Fenichel, E. P., Weinberger, D. M., Vogels, C. B. F., Brackney, D. E., Casanovas-Massana, A., et al. (2020). Pooling Saliva to Increase SARS-CoV-2 Testing Capacity. medRxiv. doi: 10.1101/2020.09.02.20183830

WEATHER AND CLIMATE IN INDONESIA | Facts and Details. Available at: http://factsanddetails.com/indonesia/Nature_Science_Animals/sub6_8a/ entry-4079.html (Accessed June 14, 2021).

Williams, E., Isles, N., Chong, B., Bond, K., Yoga, Y., Druce, J., et al. (2021). Detection of SARS-CoV-2 in Saliva: Implications for Specimen Transport and Storage. J. Med. Microbiol. 70. doi: 10.1099/jmm.0.001285

World Health Organization (2020) Diagnostic Testing for SARS-Cov-2. Available at: https://www.who.int/publications/i/item/diagnostic-testing-for-sars-cov-2.

Wyllie, A. L., Fournier, J., Casanovas-Massana, A., Campbell, M., Tokuyama, M., Vijayakumar, P., et al. (2020). Saliva or Nasopharyngeal Swab Specimens for
Detection of SARS-CoV-2. N. Engl. J. Med. 383, 1283-1286. doi: 10.1056/ nejmc2016359

Xu, R., Cui, B., Duan, X., Zhang, P., Zhou, X., and Yuan, Q. (2020). Saliva: Potential Diagnostic Value and Transmission of 2019-nCoV. Int. J. Oral. Sci. 12, 11. doi: 10.1038/s41368-020-0080-Z

Zhang, T., Cui, X., Zhao, X., Wang, J., Zheng, J., Zheng, G., et al. (2020). Detectable SARS-CoV-2 Viral RNA in Feces of Three Children During Recovery Period of COVID-19 Pneumonia. J. Med. Virol. 92, 909-914. doi: $10.1002 /$ jmv.25795

Conflict of Interest: CM, SRV, KJ, and AI are employees of Nalagenetics Pte Ltd, Singapore, holding the trademark of QuickSpit ${ }^{\mathrm{TM}}$. AI has financial holdings in Nalagenetics Pte Ltd, Singapore. CM, MMMK, SRV, and SA have filed a patent (Patent application number: S00202103063) on the RNA-Extraction-free treatment of saliva specimens for SARS-CoV-2 diagnosis.

The remaining authors declare that the research was conducted in the absence of any commercial or financial relationships that could be construed as a potential conflict of interest.

Publisher's Note: All claims expressed in this article are solely those of the authors and do not necessarily represent those of their affiliated organizations, or those of the publisher, the editors and the reviewers. Any product that may be evaluated in this article, or claim that may be made by its manufacturer, is not guaranteed or endorsed by the publisher.

Copyright $\odot 2021$ Mahendra, Kaisar, Vasandani, Surja, Tjoa, Chriestya, Junusmin, Widowati, Irwanto and Ali. This is an open-access article distributed under the terms of the Creative Commons Attribution License (CC BY). The use, distribution or reproduction in other forums is permitted, provided the original author(s) and the copyright owner(s) are credited and that the original publication in this journal is cited, in accordance with accepted academic practice. No use, distribution or reproduction is permitted which does not comply with these terms. 